\title{
Thermal and Caloric Properties of Fluids from Non-Equilibrium Molecular Dynamics Simulations Using the Two-Gradient Method $^{1}$
}

\author{
Martin P. Lautenschlaeger, Hans Hasse \\ Laboratory of Engineering Thermodynamics (LTD), University of Kaiserslautern, \\ Erwin-Schrödinger-Straße 44, 67663 Kaiserslautern, Germany
}

\begin{abstract}
Transport properties of fluids can be determined efficiently from non-equilibrium molecular dynamics simulations using the two-gradient method which was introduced recently. It is shown here that also thermal and caloric properties of fluids can be determined accurately and efficiently along with the transport properties using this method. In a single run, all these properties are obtained for a series of state points at different temperatures and constant pressure. The truncated and shifted Lennard-Jones (LJTS) fluid is studied here as a test case. Data are reported for about 700 state points in the range of $(T=[0.7,8.5]$ and $\rho=[0.2,1.0])$. Besides data on the thermal conductivity, shear viscosity, and selfdiffusion the following thermal and caloric properties were measured: pressure $p$, internal energy $u$, enthalpy $h$, isobaric heat capacity $c_{\mathrm{p}}$ and thermal expansion coefficient $\alpha_{\mathrm{p}}$. The results of the thermal and caloric properties agree very well with those from an accurate equation of state from the literature. Also the shear rate dependence of these properties can be studied easily with the two-gradient method.

Keywords: local equilibrium; Lennard-Jones fluid; isobaric heat capacity; thermal expansion coefficient
\end{abstract}

\section{Introduction}

Molecular dynamics (MD) simulations are widely used for determining material properties. But they are still computationally expensive. We have recently introduced an efficient

\footnotetext{
${ }^{1}$ The Version of Record of this manuscript has been published and is available in THE JOURNAL OF CHEMICAL PHYSICS 27 Dec 2018 https://doi.org/10.1063/1.5063985
} 
non-equilibrium molecular dynamics (NEMD) simulation method for studying transport properties of fluids. The method is characterized by a superposition of a temperature gradient $\partial T / \partial y$ and a velocity gradient $\partial \boldsymbol{v}_{x} / \partial y$, so that the thermal conductivity $\lambda$ and shear viscosity $\eta$ can be determined simultaneously [1. It is therefore called 'two-gradient method'. In addition, the self-diffusion coefficient $D$ is determined from the mean squared displacement.

The main characteristics of the two-gradient method were already discussed in detail in [1, 2]. They are only briefly summarized here: The pressure in the simulation box is constant even for large temperature gradients and velocity gradients. The magnitude of $\partial T / \partial y$ has hardly any effect on the measured properties. This was used to determine transport properties of fluids for a series of state points as a function of the temperature $T$ and at constant pressure $p$ [2]. In contrast, the magnitude of $\partial \boldsymbol{v}_{x} / \partial y$ has a significant influence on the transport properties. For small velocity gradients $\partial \boldsymbol{v}_{x} / \partial y \lesssim 0.03$ the measured transport properties do not differ from those in equilibrium. By increasing the shear rate $\dot{\gamma}=\partial \boldsymbol{v}_{x} / \partial y$ the method can be used to study the shear rate dependence of the transport properties and the pressure $p[1]$.

In the present work, thermal and caloric properties of fluids were studied using the twogradient method. Therefore, its functionality as described in [1, 2] was slightly extended inspired by the work of Matsubara et al. [3], but the main characteristics as described above were maintained. The most important difference is that also the internal energy $u$ was measured. Therefrom, together with the data on the density $\rho$ and the pressure $p$, the enthalpy $h$ was calculated. As the pressure in the simulation box is constant, also the isobaric heat capacity $c_{\mathrm{p}}$ and isobaric thermal expansion coefficient $\alpha_{\mathrm{p}}$ were determined from the data at different temperatures that are available also in a single run. The computational expenses of the additional determination of the thermal and caloric properties are negligible and essentially the same as those for the determination of only $\lambda, \eta$, and $D$, cf. [1, 2]. Thus, the two-gradient method as it is discussed in the following has the following major advantages: It is highly efficient, yields a large variety of thermodynamic properties at different state points in a single simulation run, does not require the knowledge of an equation of state (EOS) for determining derived quantities, such as $c_{\mathrm{p}}$ and $\alpha_{\mathrm{p}}$, and it can also be used to study the shear rate dependence of thermodynamic properties. 
As in the previous work [1, 2], the two-gradient method was tested here using the Lennard-Jones fluid truncated and shifted at the cut-off radius $r_{\mathrm{c}}^{*}=2.5 \sigma$ (LJTS fluid). Although this fluid is widely used for studying nano-scale processes [4, 5], to the best of our knowledge just one data set on $u$ and $h$ [6], and no data set on $c_{\mathrm{p}}$ and $\alpha_{\mathrm{p}}$ of the LJTS fluid is available in the literature. In the present work, these properties as well as $p, \lambda$, $\eta$, and $D$ were studied at about 700 thermodynamic states in the range of $T=[0.7,8.5]$ and $\rho=[0.2,1.0]$. In the present study, a large temperature gradient $(\partial T / \partial y=0.1)$ and a small velocity gradient $\left(\partial \boldsymbol{v}_{x} / \partial y=0.03\right)$ were superimposed and data on the thermal, caloric, and transport properties were measured simultaneously. The new data set on thermal and caloric properties was compared with the accurate EOS for the LJTS fluid of Thol et al. [6]. The results agree very well over the whole range of thermodynamic states studied here, which is substantially larger than the range for which the EOS of Thol et al. was thought to be valid by its developers [6]. The data on transport properties are in good agreement with previous results, see [2]. Altogether, this confirms that the hypothesis of local equilibrium [7 9] is valid over a wide range of thermodynamic states and for many different thermodynamic properties, as was also shown recently [1-3, 10, 11].

The present work also confirms that the two-gradient method is simple, robust, efficient, accurate, and flexible. Although, it was only tested for a pure simple fluid so far, its applicability is not limited to that class of fluids.

This paper is organized as follows: in Section 2 the extensions of the original twogradient method are introduced. In Section 3 the simulation setup used in the present work and the LJTS force field are specified. In Section 4 the extent of the study is described. Results of the comprehensive study are presented and compared to results from the EOS of Thol et al. [6] in Section 5. Finally, the conclusions are drawn in Section 6.

\section{Extension of the Two-Gradient Method}

\subsection{Overview}

The simulation setup is adopted from our previous studies in which the two-gradient method was introduced [1, 2]. A sketch of the simulation setup is shown in Figure 1. The system is completely filled with fluid for which the mean particle density is prescribed. It is 


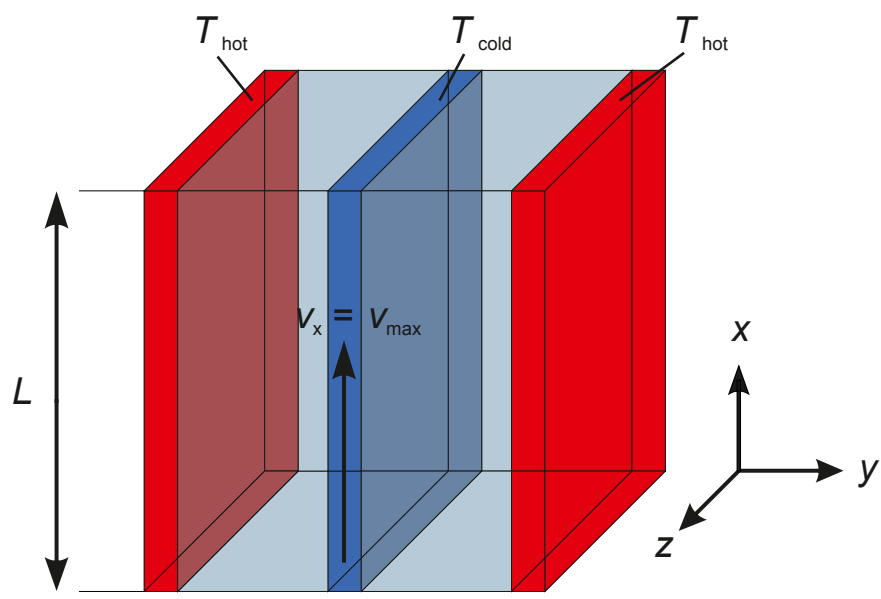

Figure 1: Scheme of the simulation setup used for the two-gradient method. The control volumes in which the temperature and the velocity are prescribed are marked in blue and red. The regions between the control volumes in which responses are recorded are unconstrained. The shear flow is in $+x$-direction. The heat flux is in $y$-direction.

symmetrical in $y$-direction, cf. Figure 1, The shear flow is in $+x$-direction. A temperature gradient is applied in $y$-direction. Periodic boundary conditions are used in all directions, such that the pressure in the box fluctuates around a constant value. There are no walls.

The following thermal, caloric, and transport properties were measured simultaneously in a single simulation run as functions of $\rho$ and $T: p, u, h, c_{\mathrm{p}}, \alpha, \lambda, \eta$, and $D$. With other NEMD methods for determining transport properties, typically only a single property is determined - at computational costs which are similar to those of the present method. This is especially remarkable when considering that due to the local equilibrium [1, 2, 7, 9] data at about 15 state points which differ in the temperature can be obtained with the present setup in a single run, whereas with competing methods typically only a single state point is investigated.

All methodological details of the two-gradient method, as they were described in [1], remain unchanged. The modifications that were necessary to study also the thermal and caloric properties are described in the following. They are mere extensions. Following [1], for all observables profiles along the $y$-direction are determined. Therefore, the simulation box is uniformly discretized in $y$-direction so that flat bins with equal thickness are obtained. In addition to the original observables, i.e. number density $\rho$, temperature $T$, velocity $\boldsymbol{v}$, heat flux $\boldsymbol{j}$, and stress tensor $\boldsymbol{\sigma}$, in the present work also the scalar properties 
$u$ and $h$ are determined in each bin via block-averaging. From the profiles, also $c_{\mathrm{p}}$ and $\alpha_{\mathrm{p}}$ can be determined as the pressure in the simulation box is constant. Details are given below for the case of pure simple fluids.

\subsection{Fundamentals}

Internal energy and enthalpy. The specific internal energy $u$ in a given bin is obtained here from Equation 1, see [12],

$$
u=\frac{1}{2 N_{\mathrm{bin}} M_{\mathrm{bin}}}\left[\sum_{i}^{N_{\mathrm{bin}}} m_{i} \boldsymbol{v}_{i, \text { therm }}^{2}+\sum_{i}^{N_{\text {bin }}} \sum_{j \neq i}^{N_{\text {Neighbor }}} \Phi\left(r_{i j}\right)\right],
$$

where $m_{i}$ is the mass of a particle $i$ and $M_{\text {bin }}$ is the total mass of a bin. $\boldsymbol{v}_{i, \text { therm }}$ is the undirected thermal velocity of a particle $i$. For details about its determination see [1]. $N_{\text {bin }}$ is the total number of particles in the bin. $N_{\text {Neighbor }}$ is the number of interacting neighbors per particle $i$. $\Phi\left(r_{i j}\right)$ is the potential energy between the particles $i$ and $j$. Therefrom, the specific enthalpy $h$ is determined:

$$
h=u+p \frac{V_{\text {bin }}}{M_{\text {bin }}}=u+p \frac{1}{\rho_{\mathrm{m}}},
$$

where $V_{\text {bin }}$ is the volume of the bin and $\rho_{\mathrm{m}}$ is the mass density.

Post-processing analysis. Just as the profiles introduced in [1], also the profiles of the new properties $u$ and $h$ are time-averaged over the production run. An example is presented in Figure 2, where the symmetry in the $y$-direction is indicated with vertical black lines, i.e. the axis origin. The thermostatted control volumes are depicted as redand blue-shaded regions. They are excluded from the analysis, because here the curvature of the profiles is affected by the thermostats and the velocity control.

Despite the symmetry, the left $(y<0)$ and the right $(y \geq 0)$ half of the system are initially treated independently. Least squares regressions are separately conducted on the left and the right side in the unconstrained regions. Second-order polynomials are fitted to the profiles of $\rho_{\mathrm{m}}, T$, and $h$. The numbers for $c_{\mathrm{p}}$ and $\alpha_{\mathrm{p}}$ are determined bin-wise as

$$
c_{\mathrm{p}}=\frac{\partial h}{\partial T}=\frac{\partial h}{\partial y}\left(\frac{\partial T}{\partial y}\right)^{-1}
$$

and

$$
\alpha_{\mathrm{p}}=-\frac{\partial\left(\ln \rho_{\mathrm{m}}\right)}{\partial T}=-\frac{\partial\left(\ln \rho_{\mathrm{m}}\right)}{\partial y}\left(\frac{\partial T}{\partial y}\right)^{-1} .
$$



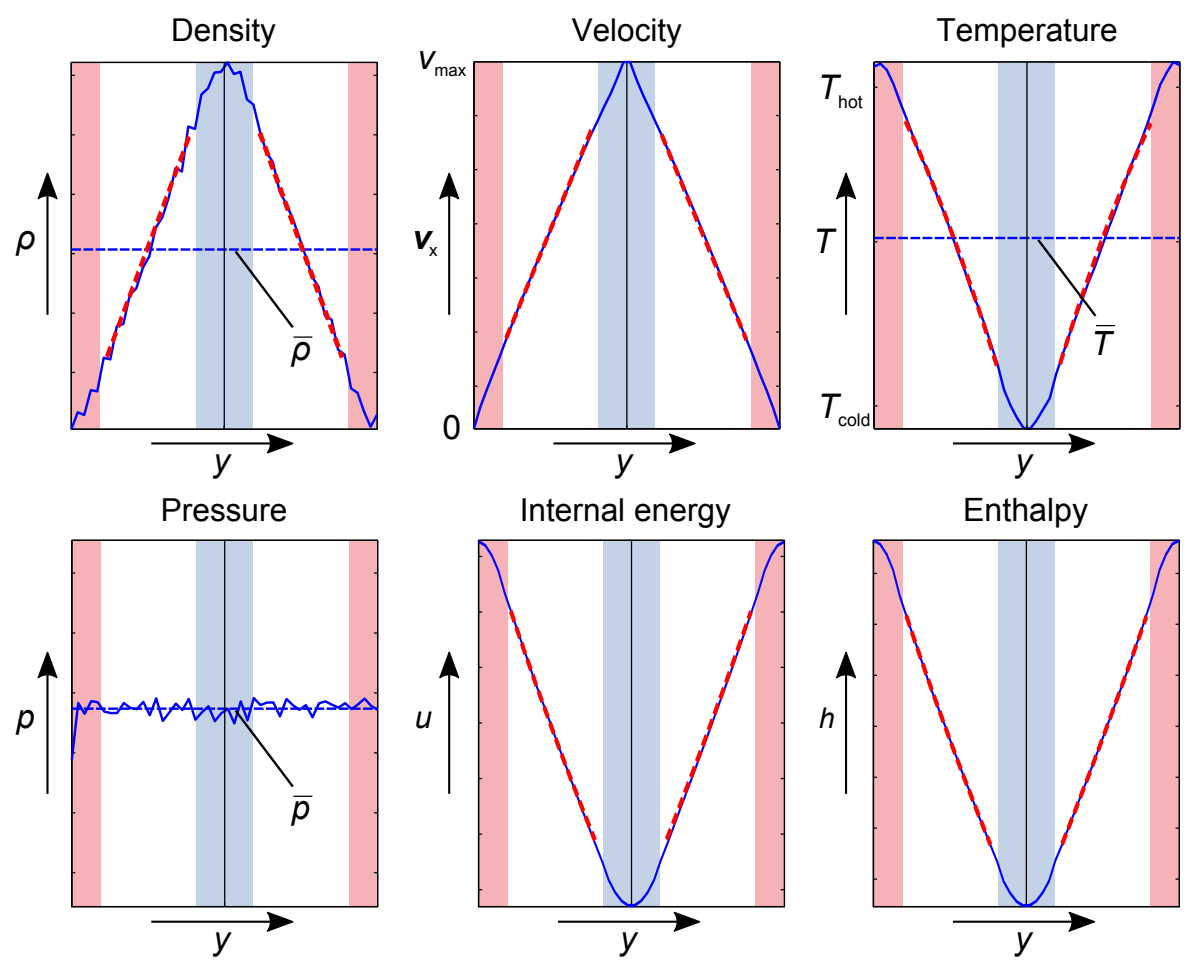

Figure 2: Overview over a selection of observables that were measured using the two-gradient method. The bin-wise results are depicted as blue solid lines. The mean values of the density $\bar{\rho}$, the temperature $\bar{T}$, and the pressure $\bar{p}$ are indicated with the vertical blue dashed lines. The regression fits are shown with the red dashed lines. They are only fitted to the profiles in the unconstrained regions between the control volumes. The latter are indicated by the shaded areas. 
All gradients in the Eqs. (3) \& (4) are also approximated bin-wise by applying the finite difference method to the regression fits.

In [1] and [2] three variants of the two-gradient method are described: the 'local method', the 'global method' and the 'shear-rate study method'. When using the global method, $u, h, c_{\mathrm{p}}$, and $\alpha_{\mathrm{p}}$ are determined in each bin and averaged along the gradients in the unconstrained regions. The averages are assigned to a single thermodynamic state point, e.g. $u(\bar{T}, \bar{\rho})$, where the bar indicates the spatial average, cf. Figure 2.

When using the local method, $u, h, c_{\mathrm{p}}$, and $\alpha_{\mathrm{p}}$ are determined in each bin. Due to the symmetry a mean value from the two corresponding opposed bins on the left and the right side is determined. The assignment to the mean thermodynamic state of each bin is done accordingly. Thus, within a single simulation run a series of thermodynamic properties is determined simultaneously.

And when using the shear rate study method, a small and perfectly linear temperature gradient is applied via a series of Gaussian isokinetic thermostats. This ensures that $\dot{\gamma}$ can be increased up to large values without a deterioration of the results, see [1]. The post-processing analysis is similar to that of the global method. The thermal and caloric properties are determined as e.g. $u(\bar{T}, \bar{\rho}, \dot{\gamma})$.

\section{Molecular Model and Simulation Setup}

Molecular model. The two-gradient method was tested using the LJTS fluid, which is based on the Lennard-Jones (LJ) potential $u^{\mathrm{LJ}}$. These potentials are known to reproduce thermo-physical properties of simple nonpolar fluids very well and are frequently applied to investigate nano-scale processes [5, 13, 15].

The LJTS potential is

$$
u^{\mathrm{LJTS}}\left(r_{i j}\right)=\left\{\begin{array}{ll}
u^{\mathrm{LJ}}\left(r_{i j}\right)-u^{\mathrm{LJ}}\left(r_{\mathrm{c}}\right), & r_{i j}<r_{\mathrm{c}} \\
0, & r_{i j} \geq r_{\mathrm{c}}
\end{array},\right.
$$

where

$$
u^{\mathrm{LJ}}\left(r_{i j}\right)=4 \varepsilon\left[\left(\sigma / r_{i j}\right)^{12}-\left(\sigma / r_{i j}\right)^{6}\right] .
$$


$\varepsilon$ is the energy parameter, describing dispersive attraction and $\sigma$ is the size parameter describing the repulsion. $r_{i j}$ is the distance between the two LJTS particles $i$ and $j$. In the present study, the cut-off radius is $r_{\mathrm{c}}^{*}=2.5 \sigma \cdot m=1$ such that the mass density $\rho_{\mathrm{m}}$ here is identical to the number density $\rho$. In the following, all observables are given in reduced units, cf. Table 2 in the Appendix.

Simulation setup. The simulation box is cubic and has the edge length $L$. Each system investigated in the present study contains about 10,000 particles. To avoid finite size effects the minimum edge length is set to $L_{\min }=24$, cf. [2]. The box is discretized with 48 bins. The corresponding bin thickness is at least $\Delta y=L / 48$. The temperature gradient and the velocity gradient are set to $\partial T / \partial y=0.1$ and $\partial \boldsymbol{v}_{x} / \partial y=0.03$, respectively. Correspondingly, temperatures and velocities are prescribed in certain control volumes (see Figure 1). In the present study, the width of each temperature control volume and each velocity control volume is $2 \sigma$ and $0.5 \sigma$, respectively. As discussed in [1], the data of the different aforementioned properties is determined in the unconstrained regions between these control volumes. In the present work, the simulation setup corresponds to the aforementioned local method which is described in [1] in more detail.

The time step is $\Delta \tau=0.002$. A simulation run is initiated by 500,000 time steps of equilibration in which a steady state is established, i.e. a stable temperature and velocity gradient are built up. Subsequently, the production run consists of 2,000,000 time steps. Here, block-averaging is applied over 10,000 time steps each to determine the mean standard deviation for all observables. The post-processing steps are accounted for by error propagation. This error analysis is done for each data point. The averaging of the mean standard deviations over a set of data points is called 'mean estimated uncertainty' in the following. The error analysis is adopted from our previous work, for details see [1].

All simulations are carried out with a substantially extended version of the MD code ls1 mardyn [16].

\section{Overview of the Study}

Figure 3 gives an overview of the present study. $p, u, h, c_{\mathrm{p}}$, and $\alpha_{\mathrm{p}}$ were determined for 679 state points as a function of $T$ and $\rho$ for a wide range of thermodynamic states $(T=[0.7,8.5]$ and $\rho=[0.2,1.0])$. The mean estimated uncertainty of the full data set for 


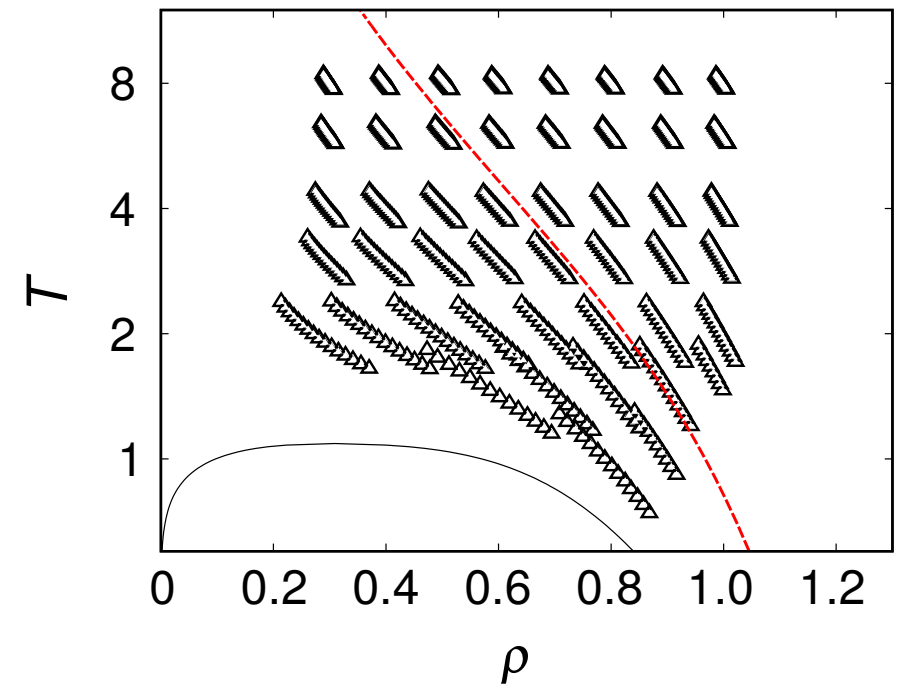

Figure 3: Overview of the present study. The investigated state points are shown in a $T, \rho$-plot. Each series of symbols represents a series of about 15 state points that were determined within a single simulation run. The red dashed line is the isobar $p=6.8$ as determined from the EOS of Thol et al. [6]. It indicates the upper limit of the validity range of this EOS.

$\left(p, u, h, c_{\mathrm{p}}\right.$, and $\left.\alpha_{\mathrm{p}}\right)$ is $(1.2 \%, 3.1 \%, 1.9 \%, 2.6 \%$, and $2.4 \%)$. As mentioned before, $(\lambda, \eta$, and $D$ ) are evaluated from the same simulations with the mean estimated uncertainties $(0.9 \%, 2.5 \%$, and $5.7 \%)$, respectively.

The numerical results are reported in the Supporting Information. No data are reported for $\rho<0.2$ as the present simulation method is not recommended for low densities [1].

\section{Thermal and Caloric Properties of the LJTS Fluid}

To the best of our knowledge there is no data set on $c_{\mathrm{p}}$ and $\alpha_{\mathrm{p}}$ of the LJTS fluid available in the literature. For $u$ and $h$ there is only one which is from Thol et al. 6]. It consists of data for about 700 state points in the gaseous, liquid, and supercritical regime. Based on that data the authors developed an fundamental EOS from which any equilibrium thermal and caloric property of the LJTS fluid can be derived. The calculation for most of these properties is implemented in the open-source software TREND 3.0 [17], which was used for the evaluation of the results here. The EOS of Thol et al. [6] is valid for the temperature range $T=[0.64,11.0]$ and the pressure range $p<6.8$ according to its developers [6]. This corresponds to the space below the red dashed line in Figure 3.

In the following, results from the present study for $p, u, h, c_{\mathrm{p}}$, and $\alpha_{\mathrm{p}}$ as functions of 
$T$ and $\rho$ are compared to the results from the EOS of Thol et al. [6]. The data from the present work is grouped in 'series', where each series stems from a single simulation run and is for constant pressure.

In Figure 4 selected data for all studied properties are shown as a function of the density. Different data series from the present work do not have exactly the same pressure, as the pressure is not predefined. Hence for the comparisons in Figure 4 data sets were chosen that have similar pressures. They are compared to results from the EOS of Thol et al. [6] for which pressure bands are given (lines): $p=3.9 \pm 5 \%$ (blue squares), $p=6.0 \pm 5 \%$ (green diamonds), and $p=18.0 \pm 5 \%$ (red circles). The results from the present study and from the EOS of Thol et al. [6] agree very well for all properties.

In Figure 5 the whole data set is compared to the results from the EOS of Thol et al. [6] in deviation plots. The color indicates the mean temperature of the respective data series. The simulation values from the present work agree very well with the results from the EOS of Thol et al. [6] with deviations below $\pm 5 \%$. Slight systematic deviations are only observed for some data series for $c_{\mathrm{p}}$ and $\alpha_{\mathrm{p}}$ for $(\bar{T}=1.0, \bar{\rho}=0.8)$ and $(\bar{T}=1.5, \bar{\rho}=0.6)$, that are closest to the vapor-liquid coexistence line.

The best agreement is observed for the data of $p$. This is particularly important as it underpins the hypothesis of local equilibrium [7 7 throughout all simulations from the present study.

Additionally, deviations between the simulation data and the results from the EOS of Thol et al. [6] are quantified using the average absolute deviation (AAD)

$$
\mathrm{AAD}=\frac{1}{N} \sum_{i=1}^{N}\left|1-\frac{x_{i}}{x_{i, \mathrm{EOS}}}\right|
$$

and the bias

$$
\operatorname{bias}=\frac{1}{N} \sum_{i=1}^{N}\left(1-\frac{x_{i}}{x_{i, \mathrm{EOS}}}\right) .
$$

Here, $x_{i}$ stands for the property $p, u, h, c_{\mathrm{p}}$, or $\alpha_{\mathrm{p}}$ at a state $i$ determined using the twogradient method. $x_{i, \mathrm{EOS}}$ is the corresponding value from the EOS of Thol et al. [6]. $N$ is the number of thermodynamic states considered for the comparison, i.e. $N=679$ here. The numbers for AAD and bias are listed in Table 1. 

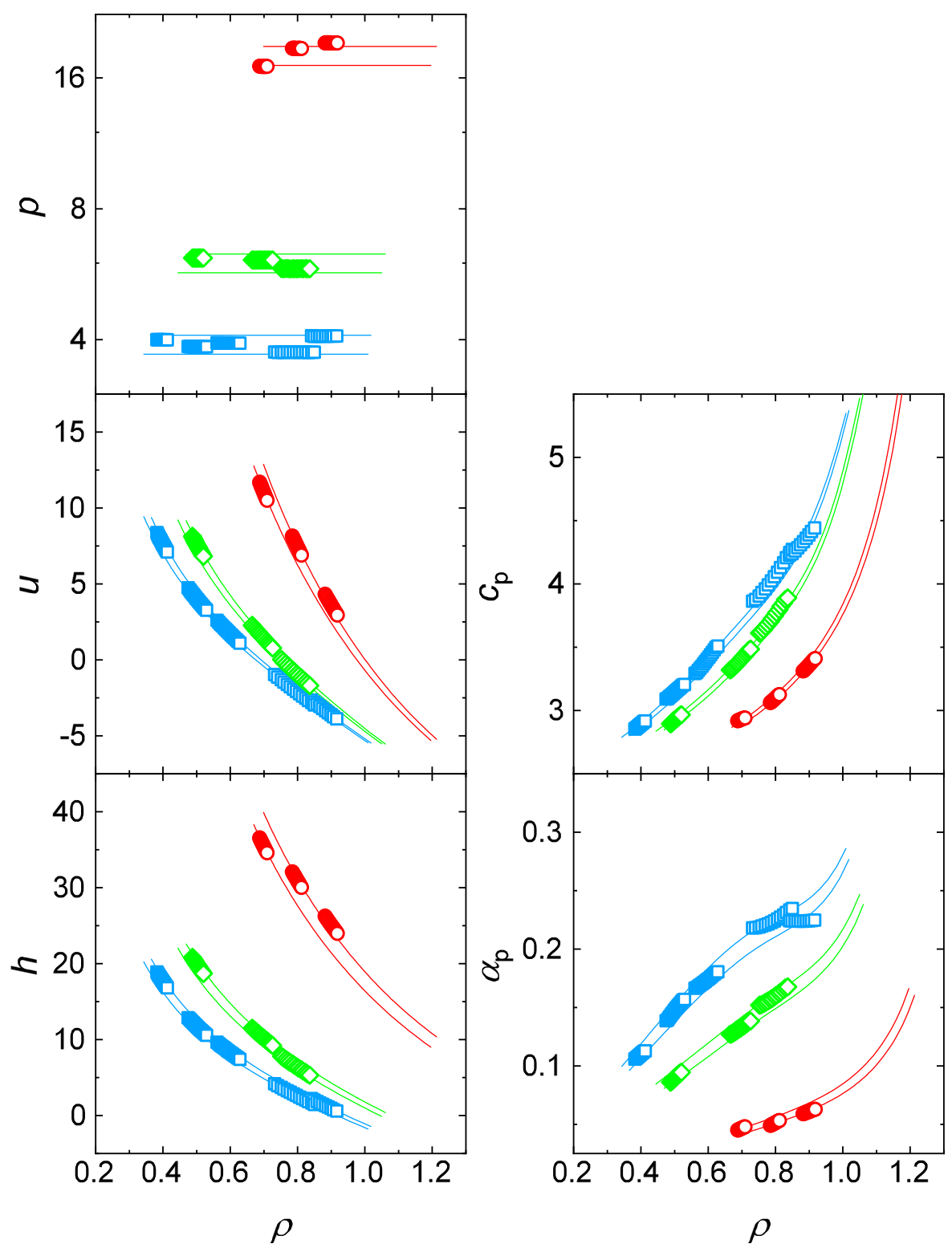

Figure 4: Thermal and caloric properties of the LJTS fluid. Data from the present work are shown in the vicinity of three isobars: $p=3.9 \pm 5 \%$ (blue squares), $p=6.0 \pm 5 \%$ (green diamonds), and $p=18.0 \pm 5 \%$ (red circles). The upper and the lower bound of the isobars are determined with the EOS of Thol et al. [6] and depicted with lines. The estimated uncertainties of the simulation data are within the symbol size. 


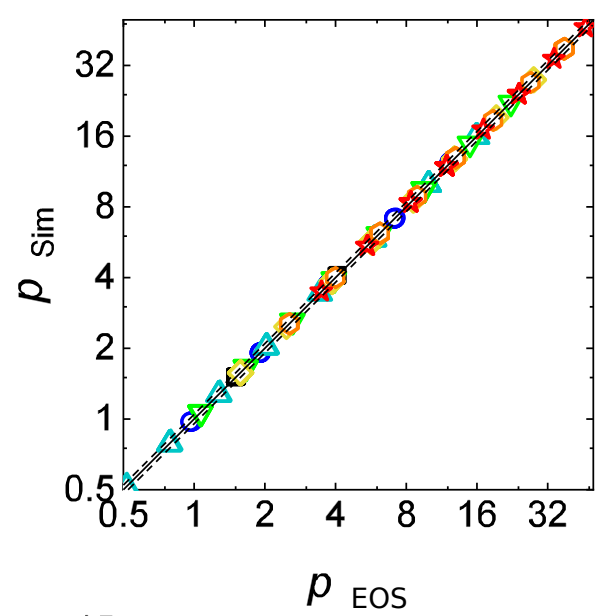

$$
\begin{aligned}
& \square \bar{T}=1.00 \\
& \circ \bar{T}=1.50 \\
& \triangle \bar{T}=2.00 \\
& \nabla \bar{T}=3.00 \\
& \diamond \bar{T}=4.00 \\
& \triangleright \bar{T}=6.00 \\
& \varangle \bar{T}=8.00
\end{aligned}
$$
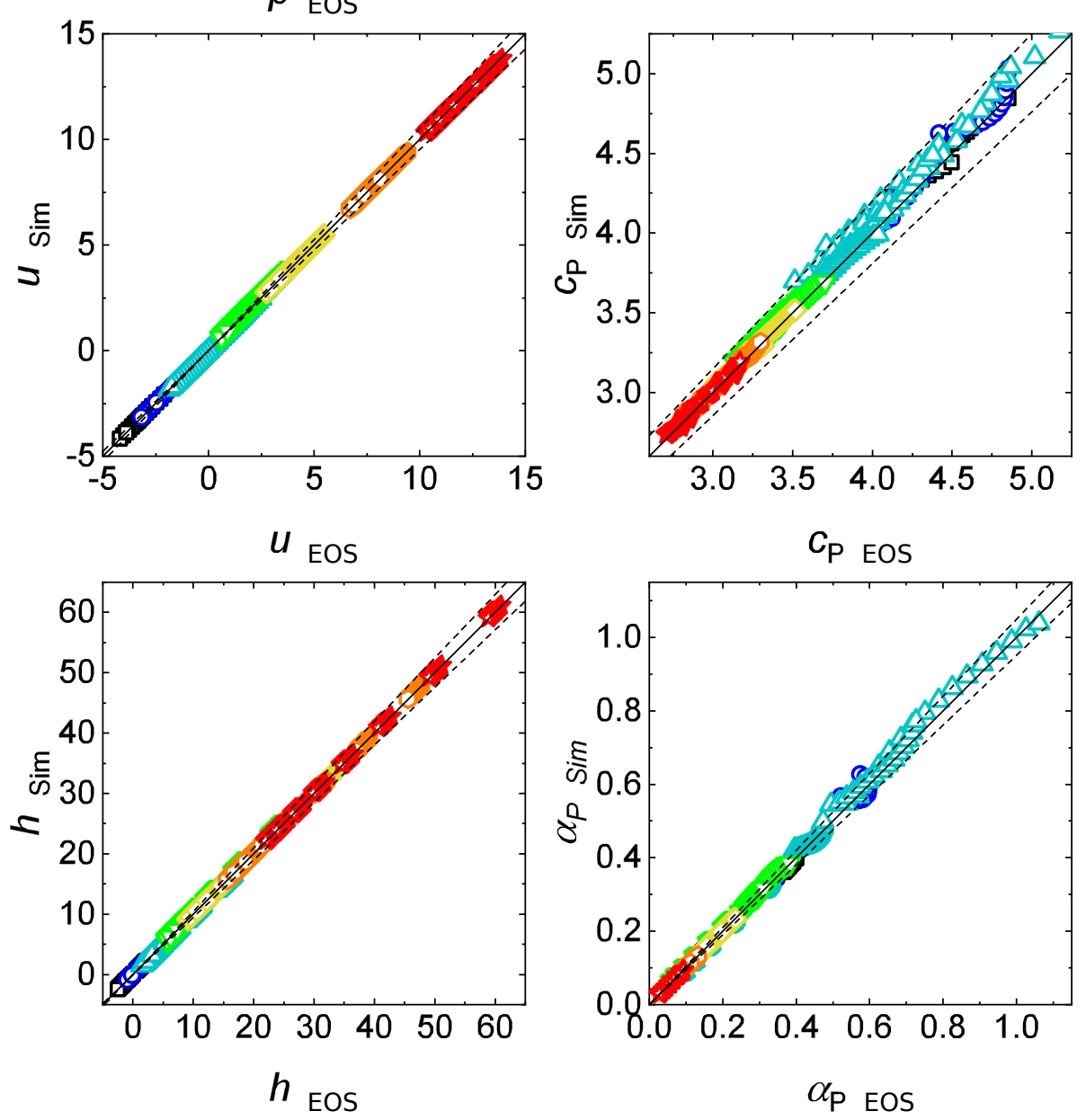

Figure 5: Deviation plots comparing the results from simulations from the present work (Sim) with those from the EOS of Thol et al. [6] (EOS). The whole data set $N=679$ thermodynamic states is shown. The dashed lines indicate a band of $\pm 5 \%$ around the solid line. The estimated uncertainty of the results from the present work is within the symbol size. The different colors indicate the mean temperature of the data sets determined in the individual simulation runs. 
Table 1: Statistical evaluation of all results from the presents work by comparison with the results from the EOS of Thol et al. [6]. As reference values, the mean estimated uncertainties (MEU) of the each property are given

\begin{tabular}{cccc}
\hline & AAD (\%) & bias (\%) & MEU (\%) \\
\hline$p$ & 0.22 & -0.187 & 1.2 \\
$u$ & 0.56 & -0.026 & 3.1 \\
$h$ & 0.34 & -0.153 & 1.9 \\
$c_{\mathrm{p}}$ & 0.81 & -0.828 & 2.6 \\
$\alpha_{\mathrm{p}}$ & 1.06 & -0.684 & 2.4 \\
\hline
\end{tabular}

The agreement between the simulation data from the present work and the results from the EOS of Thol et al. [6] is excellent for all studied properties. The deviations are generally below the statistical error of the simulation data, i.e. the mean estimated uncertainty. The bias is well below the AAD for $p, u$, and $h$. For $c_{\mathrm{p}}$ and $\alpha_{\mathrm{p}}$ the bias is somewhat larger and in the range of the AAD.

Supplementary, the simulation results of $\lambda, \eta$, and $D$ are compared to the correlations from our previous study [2]. Both data sets agree well. Thus, the data of the transport properties which are a secondary product from the present work also agree well with results from the literature, cf. Table 3 in the Appendix.

These findings are very favorable, especially when considering that a wide range of thermodynamic states was studied here. Furthermore, with the local method many different thermodynamic properties $\left(p, u, h, c_{\mathrm{p}}, \alpha_{\mathrm{p}}, \lambda, \eta, D\right)$ were determined in the present work for about 15 different state points in a single simulation run.

As a side-effect, the results from the present study indicate that the EOS of Thol et al. [6] extrapolates very well beyond the validity range $(p \leq 6.8)$ that was reported by its developers [6], cf. Figure 3, The simulation results from the present work and the results from the EOS of Thol et al. [6] agree very well in the whole range of the thermodynamic states studied here, i.e. for pressures up to $p=46.5$. 


\section{Conclusion}

In previous studies of our group [1, 2], the two-gradient method was developed and applied to study transport properties of fluids by non-equilibrium molecular dynamics simulations. The two-gradient was shown to be efficient and accurate. In the present study, this method was extended and used also to determine thermal and caloric properties, namely: pressure $p$, internal energy $u$, enthalpy $h$, isobaric heat capacity $c_{\mathrm{p}}$ and thermal expansion coefficient $\alpha_{\mathrm{p}}$ as a function of temperature and density. In addition, data on the thermal conductivity $\lambda$, shear viscosity $\eta$, and self-diffusion coefficient $D$ were determined. Due to the superposition of a large temperature gradient $(\partial T / \partial y=0.1)$ with a small velocity gradient $\left(\partial \boldsymbol{v}_{x} / \partial y=0.03\right)$, all aforementioned properties were obtained from a single simulation run for a series of state points at different temperatures and constant pressure. The hypothesis of local equilibrium is valid, such that the reported data should be considered as equilibrium values.

The two-gradient method was applied to the Lennard-Jones truncated and shifted (LJTS) fluid. Data on thermal, caloric and transport properties were determined at 679 state points which cover a wide range of thermodynamic states $(T=[0.7,8.5]$ and $\rho=[0.2,1.0])$. The simulation data were compared to results from the equation of state (EOS) of Thol et al. [6] and excellent agreement was found in the entire range that was studied here. The results from the present study show that the two-gradient method is not only applicable for the determination of transport properties but also for determining thermal and caloric properties in the same simulation run. Besides, it was shown that the EOS of Thol et al. [6], whose validity range was originally reported to be only for $p \leq 6.8$, extrapolates well to at least $p \leq 46.5$.

Altogether, a comprehensive data base for the LJTS fluid is provided. In total almost 5500 data points were determined from only 47 simulation runs. Previously, there was only one data set of $u$ and $h$ of the LJTS fluid in the literature and no data on $c_{\mathrm{p}}$ and $\alpha_{\mathrm{p}}$, so that the present study extends the available simulation data on thermal and caloric properties of the LJTS fluid significantly. More than 2000 data points for $\lambda$, $\eta$, and $D$ were determined. They are in good agreement with previous results, see [2].

The present study has proven that the two-gradient method is a promising approach 
for the accurate and efficient calculation of a variety of thermodynamic properties. The two-gradient method is particularly suited for studying also the shear rate dependence of the aforementioned thermodynamic properties. This will be the scope of future work of our group. 


\section{Appendix}

\subsection{Reduced Units}

Table 2: Definition of the physical quantities in reduced units (marked by the asterisk). The corresponding observable carrying a dimension is plain. $M$ is the mass of a single particle. $k_{\mathrm{B}}$ is the Boltzmann's constant

\begin{tabular}{|c|c|}
\hline Length & $L=\frac{L^{*}}{\sigma}$ \\
\hline Time & $\tau=\frac{\tau^{*}}{\sigma \sqrt{M / \varepsilon}}$ \\
\hline Mass & $m=\frac{m^{*}}{M}$ \\
\hline Velocity & $\boldsymbol{v}_{k}=\frac{\boldsymbol{v}_{k}^{*}}{\sqrt{\varepsilon / M}}$ \\
\hline Shear rate & $\dot{\gamma}=\frac{\dot{\gamma}^{*}}{\sqrt{\varepsilon / M} / \sigma}$ \\
\hline Density & $\rho=\rho^{*} \sigma^{3}$ \\
\hline Temperature & $T=\frac{T^{*}}{\varepsilon / k_{\mathrm{B}}}$ \\
\hline Pressure & $p=\frac{p^{*}}{\varepsilon / \sigma^{3}}$ \\
\hline Internal energy & $u=\frac{u^{*}}{\varepsilon}$ \\
\hline Enthalpy & $h=\frac{h^{*}}{\varepsilon}$ \\
\hline Heat capacity & $c_{\mathrm{p}}=\frac{c_{\mathrm{p}}^{*}}{k_{\mathrm{B}} / M}$ \\
\hline Thermal expansion coeff. & $\alpha_{\mathrm{p}}=\frac{\alpha_{\mathrm{p}}^{*}}{k_{\mathrm{B}} / \varepsilon}$ \\
\hline
\end{tabular}

\subsection{Transport Properties}

Table 3: Comparison of the data set of $\lambda, \eta$, and $D \rho$ for the LJTS fluid with the corresponding correlations from our previous study [2]. The data was determined at $N=679$ state points in the range of $(T=[0.7,8.5]$ and $\rho=[0.2,1.0])$. Numerical results are given in the Supporting Information. As reference values, the mean estimated uncertainties (MEU) of the each property are given

\begin{tabular}{cccc}
\hline & AAD (\%) & bias (\%) & MEU (\%) \\
\hline$\lambda$ & 2.34 & -1.217 & 0.9 \\
$\eta$ & 4.92 & -4.261 & 2.5 \\
$D \rho$ & 4.10 & 3.441 & 5.7 \\
\hline
\end{tabular}




\section{Conflicts of interest}

There are no conflicts to declare.

\section{Acknowledgement}

The authors gratefully acknowledge financial support by the DFG within IRTG 2057 Physical Modeling for Virtual Manufacturing Systems and Processes. The simulations were carried out on the elwe at Regional University Computing Center Kaiserslautern (RHRK) under the grant TUKL-TLMV as well as on the SuperMuc at Leibniz Supercomputing Centre (LRZ) Garching under the grant SPARLAMPE (pr48te). The present research was conducted under the auspices of the Boltzmann-Zuse Society for Computational Molecular Engineering (BZS). 


\section{References}

[1] M. P. Lautenschlaeger, M. Horsch, H. Hasse, Simultaneous determination of thermal conductivity and shear viscosity using two-gradient non-equilibrium molecular dynamics simulations, Molecular Physics 117 (2) (2019) 189-199. doi:10.1080/ 00268976.2018 .1504134 .

[2] M. P. Lautenschlaeger, H. Hasse, Transport Properties of the Lennard-Jones Truncated and Shifted Model Fluid from Non-Equilibrium Molecular Dynamics Calculations, Fluid Phase Equilibria 482 (2019) 38 - 47. doi:https://doi.org/10.1016/ j.fluid.2018.10.019.

[3] H. Matsubara, G. Kikugawa, T. Bessho, S. Yamashita, T. Ohara, Non-equilibrium molecular dynamics simulation as a method of calculating thermodynamic coefficients, Fluid Phase Equilibria 421 (2016) 1-8. doi:10.1016/j.fluid.2016.03.019.

URL http://dx.doi.org/10.1016/j.fluid.2016.03.019

[4] C. Hoheisel, Bulk viscosity of model fluids. A comparison of equilibrium and nonequilibrium molecular dynamics results, The Journal of Chemical Physics 86 (4) (1987) 2328-2333. doi:10.1063/1.452131

URL http://link.aip.org/link/JCPSA6/v86/i4/p2328/s1\{\&\}Agg=doihttp: //aip.scitation.org/doi/10.1063/1.452131

[5] J. P. Ewen, D. M. Heyes, D. Dini, Advances in nonequilibrium molecular dynamics simulations of lubricants and additives, Friction (Md) (2018) 10.1007/s40544-0180207-9. doi:10.1007/s40544-018-0207-9.

URL http://link .springer .com/10.1007/s40544-018-0207-9

[6] M. Thol, G. Rutkai, R. Span, J. Vrabec, R. Lustig, Equation of State for the LennardJones Truncated and Shifted Model Fluid, International Journal of Thermophysics 36 (1) (2015) 25-43. doi:10.1007/s10765-014-1764-4.

URL http://link .springer .com/10.1007/s10765-014-1764-4

[7] A. Tenenbaum, G. Ciccotti, R. Gallico, Stationary nonequilibrium states by molecular 
dynamics. Fourier's law, Physical Review A 25 (5) (1982) 2778-2787. doi:10.1103/ PhysRevA.25.2778.

[8] A. Tenenbaum, Local equilibrium in stationary states by molecular dynamics, Physical Review A 28 (5) (1983) 3132-3133.

[9] S. de Groot, P. Mazur, Non-Equilibrium Thermodynamics, Dover Publication, New York, 1984.

[10] F. Bresme, J. Armstrong, Note: Local thermal conductivities from boundary driven non-equilibrium molecular dynamics simulations, The Journal of Chemical Physics 140 (1) (2014) 016102. doi:10.1063/1.4858434.

URL http://www.ncbi.nlm.nih.gov/pubmed/24410242http://aip.scitation. org/doi/10.1063/1.4858434

[11] F. Bresme, Non-equilibrium simulations of fluids under thermal gradients: local properties and coupling effects, in: Joint European Thermodynamics Conference, Vol. 1, 2017, pp. 25-28.

[12] D. J. Evans, G. P. Morriss, Statistical Mechanics of Nonequilibrium Liquids, no. 1, Cambridge University Press, 2008. doi:10.1017/CB09780511535307.

URL http://ebooks . cambridge .org/ref/id/CB09780511535307

[13] M. P. Allen, D. J. Tildesley, Computer simulation of liquids, Oxford Science Publications, New York, 1989.

[14] K. M. Dyer, B. M. Pettitt, G. Stell, Systematic investigation of theories of transport in the Lennard-Jones fluid, The Journal of Chemical Physics 126 (3) (2007) 034502. doi:10.1063/1.2424714.

URL http://aip.scitation.org/doi/10.1063/1.2424714

[15] G. Rutkai, M. Thol, R. Span, J. Vrabec, How well does the Lennard-Jones potential represent the thermodynamic properties of noble gases?, Molecular Physics 115 (9-12) (2017) 1104-1121. doi:10.1080/00268976.2016.1246760.

URL https://www.tandfonline.com/doi/full/10.1080/00268976.2016. 1246760 
[16] C. Niethammer, S. Becker, M. Bernreuther, M. Buchholz, W. Eckhardt, A. Heinecke, S. Werth, H.-J. Bungartz, C. W. Glass, H. Hasse, J. Vrabec, M. Horsch, ls1 mardyn : The Massively Parallel Molecular Dynamics Code for Large Systems, Journal of Chemical Theory and Computation 10 (10) (2014) 4455-4464. doi:10.1021/ct500169q.

URL http://pubs .acs .org/doi/abs/10.1021/ct500169q

[17] R. Span, T. Eckermann, S. Herrig, S. Hielscher, A. Jäger, M. Thol, TREND 3.0., TREND. Thermodynamic Reference and Engineering Data 3.0, Lehrstuhl für Thermodynamik, Ruhr-Universität Bochum. (2016). 